\title{
Active seismic monitoring
}

\author{
R.A. Lynch Institute of Mine Seismology, Australia
}

\section{Abstract}

The use of controlled sources of seismic radiation can facilitate a better understanding of the rock mass response to mining. Ideally, the seismic sources should be highly repeatable so that differences in seismic transmission over time can be more easily studied and related to mining. Field trials of such a seismic source at El Teniente Mine (Chile) and Mponeng Mine (South Africa) have shown that massive stacking can be used to provide useful signal-to-noise ratios despite normal mining noises, on hourly time scales. The maximum range tested here is $200 \mathrm{~m}$ between source and receiver, using a 5 hour data set. Seismic velocity variations can be estimated on an hourly basis, to an accuracy of not less than $10^{-4}$. The technique appears sensitive enough to detect changes in stress caused by blasting $100 \mathrm{~m}$ away from the source-sensor ray. Significant $S$-wave radiation has also been recorded from the isotropic seismic source, and is most likely the result of $P$-S conversion at the borehole wall. The limitations of analogue cable length between source and sensor, as well as the awkwardly large datasets, have been overcome by an automatic pre-stacking algorithm within the seismic station, as demonstrated at Ridgeway mine (Australia). Pre-stacking reduces the amount of recorded data by a factor 300 , to about $80 \mathrm{MB} /$ sensor/day.

\section{Introduction}

Measurement of stress changes due to mining is a fundamental goal of geotechnical monitoring. Laboratory measurements in the 1960s showed that the seismic velocity of dry or partially saturated rock varies in response to changes in applied stress (e.g. Birch 1960, 1961; Scholz, 1968; Nur and Simmons, 1969). These experiments used confining stresses of a few MPa, corresponding to typical mining stresses at a few kilometers depth. This effect has been theoretically modelled (e.g. Hudson, 1981; Boadu and Long, 1996). The stress-velocity coupling is attributed to opening (closing) of micro-cracks due to decreasing (increasing) pressures normal to the crack surface (e.g. Walsh, 1965; Nur, 1971; Crampin and Zatsepin, 1997).

Stress-velocity coupling coefficients in the range $10^{-6} / \mathrm{Pa}$ to $10^{-9} / \mathrm{Pa}$ have been reported (De Fazio et al., 1973; Reasenberg and Aki, 1974; Gladwin, 1977; Leary et al., 1979; Yukutake et al., 1988; Sano et al., 1999; Yamamura et al., 2003; Silver et al., 2007; Niu et al., 2008) with the smaller values corresponding to more massive rock at deeper experimental sites. For typical mines, it seems reasonable to assume a value of $10^{-8} / \mathrm{Pa}$, which implies we must be able to measure seismic velocity changes of the order of $0.001 \%$ in order to detect a mining-induced stress change of the order of $1 \mathrm{kPa}$.

These previous studies have measured volumes of rock with static fracture densities: the applied stress variations have not resulted in the formation of new fractures, only the opening/closing of existing microcracks. Of course, in mines the stress changes are large enough to cause fresh fractures. Increases in fracture density result in a decrease in seismic velocities. In particular, the S-wave velocity drops by $1 \%$ for each $1 \%$ increase in the crack density in the low density limit (Boadu and Long, 1996). In the skin of excavations, the velocity drop is of the order of $1 \%$, while in caving mines this effect is of the order of $10 \%$. In the short time scales of mining, fractures are not healed, only created. This means that seismic velocities would only decrease in response to mining-induced fracturing.

Attempts to make in situ measurements of seismic velocities to high precision have been reported using vibrator (De Fazio et al., 1973), air-gun (Reasenberg and Aki, 1974; Leary et al., 1979), magnetostrictive (Gladwin, 1977) and piezoelectric (Yukutake et al., 1988; Sano et al., 1999; Yamamura et al., 2003; Silver et al., 2007; Niu et al., 2008) sources of repeatable, controlled seismic radiation. 
The latest studies, based on piezoelectric sources, have used rather short $(\sim 10 \mathrm{~m})$ baselines between source and sensor and utilised massive stacking to boost signal-to-noise ratios in order to extract tiny velocity variations from cross-correlations. Recent work (Lynch, 2010) at El Teniente underground copper mine in Chile demonstrated that such techniques can be successful over a range of $137 \mathrm{~m}$ despite the high noise of normal mining operations. An accuracy of velocity variation measurement of the order of $0.004 \%$ was estimated in that study.

It seems possible to obtain continuous measurements of stress, via the proxy of seismic velocity, in a working mine. The benefits of this would include better calibration of numerical stress models and even the promise of more accurate indicators of potential rock mass instabilities (Niu et al., 2008). However, the relative advantages of this technique for stress measurement compared with high accuracy strain measurements (e.g. Ogasawara et al., 2002) can be debated.

There are two primary advantages of high precision seismic velocity measurements over traditional strain meters. Firstly, seismic velocity measurements are made over a zone, unlike the point strain measurements. Secondly, certain kinds of seismic sources produce both P- and S-waves, and if these velocities can be measured simultaneously then an effective medium (Young's modulus and Poisson's ratio) can be estimated. These advantages make the technique very useful as a geotechnical monitoring tool. As an example, it would be possible (and practical) to monitor a regional pillar in the mine and estimate its effective strength as a function of time. Another example would be the ability to monitor the rock above a cave in order to better track the cave front advance with time.

Lynch (2010) proposed Active Seismic Monitoring. This is a concept in which a few controlled repeatable seismic sources are added to the standard passive seismic monitoring system, which is currently used routinely by over 200 mines around the world. The active seismic measurements would take place continuously - at the same time as, and without interruption to, normal passive seismic monitoring. The standard sensor array would be used as receivers, and the range would be practical enough for mine-scale problems.

For this technique to become routinely available, the key issues of range and data processing require attention. The El Teniente experiment demonstrated a range of $140 \mathrm{~m}$. To extend this range, an experiment at the Mponeng deep gold mine in South Africa was performed, and this is presented in Section 2. In that measurement, the necessary data processing was rather cumbersome and so a data reduction method called pre-stacking was implemented in a new experiment at Ridgeway Mine in Australia, and is discussed in Section 3.

\section{$2 \quad$ Experiment at Mponeng Mine, South Africa}

A piezoelectric source was installed in a $10 \mathrm{~m}$ water-filled vertical borehole at Mponeng Mine's SARS test site, at distances of $107 \mathrm{~m}$ and $206 \mathrm{~m}$ from triaxial $2.3 \mathrm{kHz}$ accelerometers. This part of the mine is about $3,100 \mathrm{~m}$ below surface. A plan view of this arrangement is shown in Figure 1. 


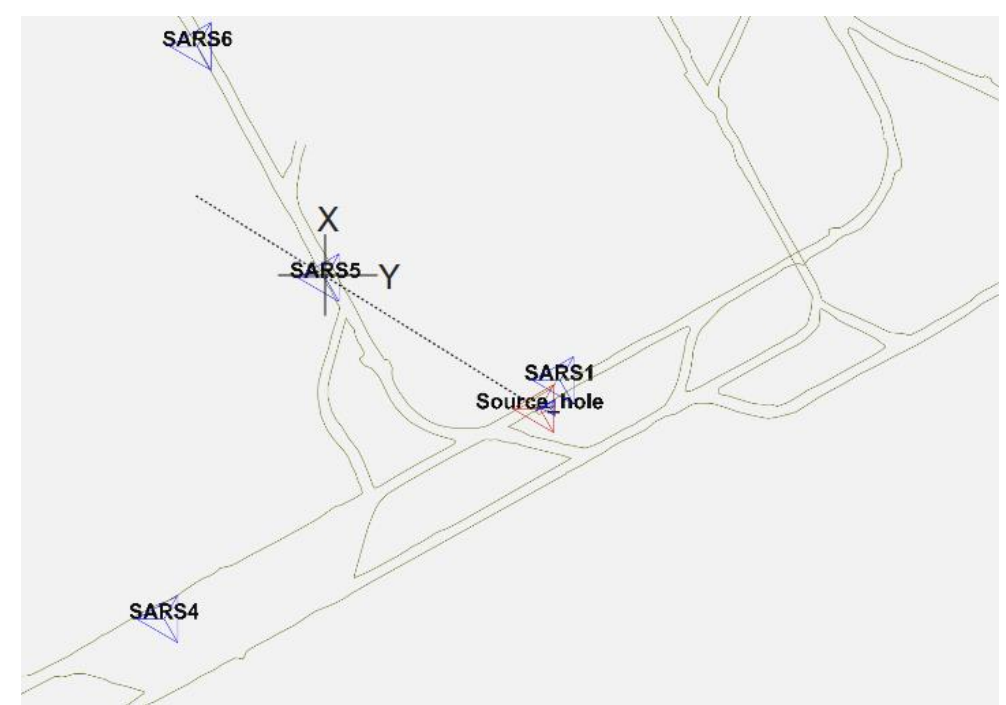

Figure 1 Plan view of the SARS test site at Mponeng mine. A piezoelectric source was installed $5 \mathrm{~m}$ away from sensor SARS1 at distances of $107 \mathrm{~m}$ and $206 \mathrm{~m}$ from SARS5 and SARS6, respectively

A vertical geophone was dangled into the water column about $1 \mathrm{~m}$ above the source to record the source pulse. This sensor was connected to a channel of the GS seismic station, together with the $X$ - and Y-components of SARS5. These three channels were sampled at $24 \mathrm{kHz}$ and continuous seismogram data recorded to disk. The source was pulsed 3 times per second. While the peak-to-peak voltage is rather high $(1,600 \mathrm{~V})$, the duty cycle of around $0.3 \%$ means the portable source driver only consumes around $6 \mathrm{~W}$ of continuous power. The pulse central frequency was $1,600 \mathrm{~Hz}$. The source pulse, as recorded on the vertical geophone, is shown in Figure 2.

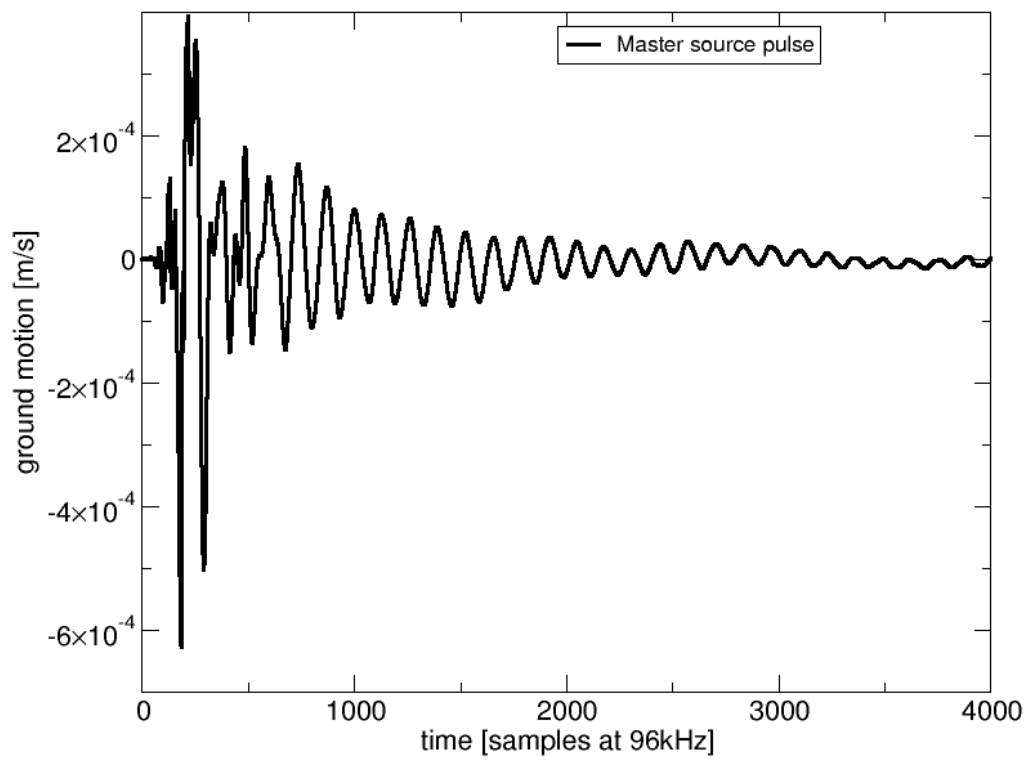

Figure 2 The source pulse recorded on the vertical geophone dangled in the water column about $1 \mathrm{~m}$ above the source. The pulse (first part of the seismogram) excites a resonance in the water column - the $720 \mathrm{~Hz}$ harmonic signal in the coda of this seismogram. Note this seismogram has been up-sampled by a factor 4 to produce $96 \mathrm{kHz}$ from the native $24 \mathrm{kHz}$ recording

Each pulse uses about $2 \mathrm{~J}$ of electrical power, and so the radiated seismic energy is very low - roughly equivalent to a magnitude -4 event. This means that the signal at distant sensors is very weak, and buried deep in the noise. As discussed in Lynch (2010), massive stacking is used to retrieve the signal. If the remote sensor's seismograms are time-aligned with each source pulse, then the consistent parts of the signal (the 
source pulse arriving at that remote sensor) remains the same during averaging, while the random uncorrelated noise is reduced. In this way the signal-to-noise ratio (SNR) grows in a statistical $(\sqrt{N})$ manner as $\mathrm{N}$ seismograms are stacked. An example of how stacking works with this data is presented in Figure 3.
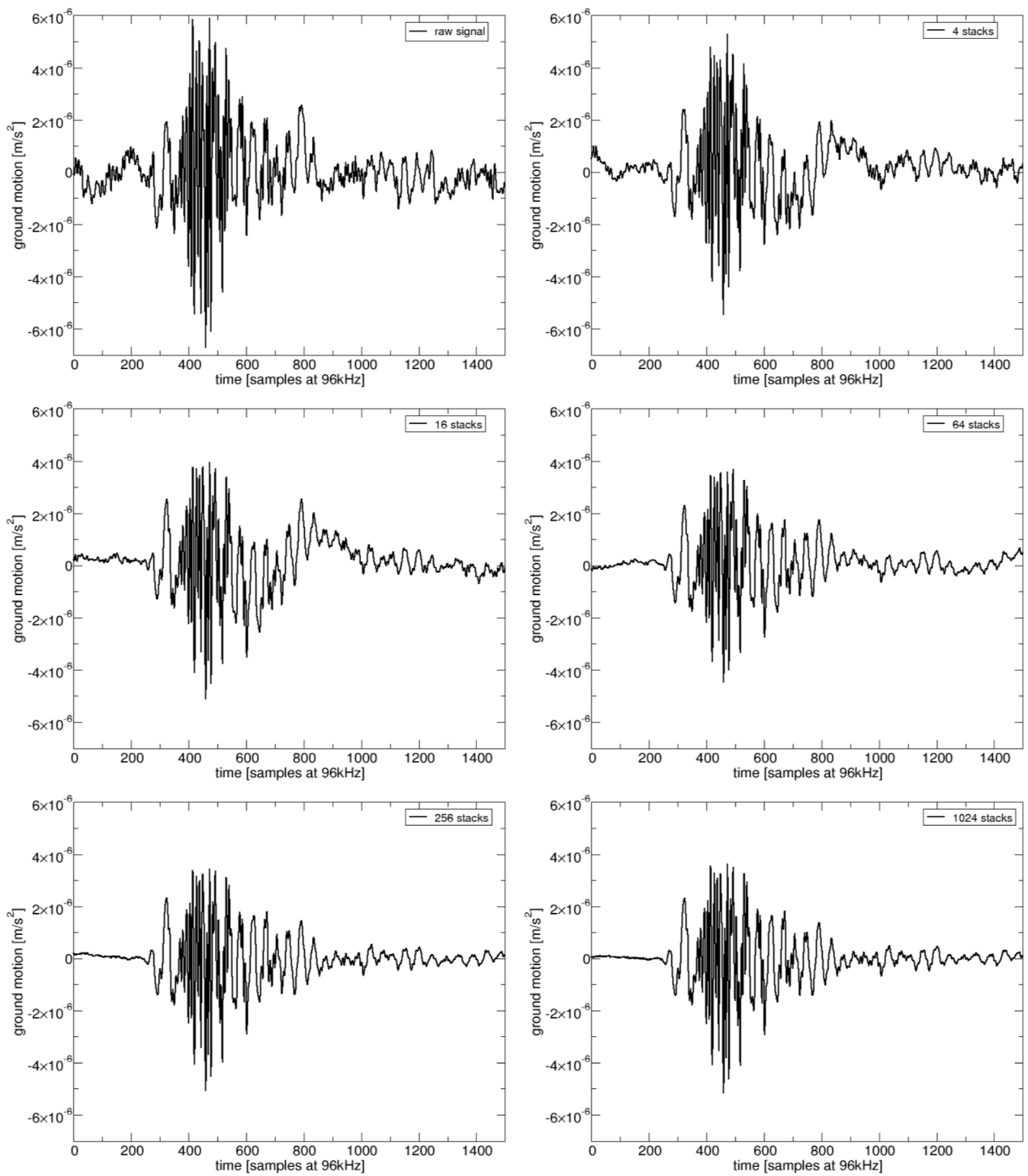

Figure 3 Example of how stacking operates on the recorded signals to reduce the level of uncorrelated random noise while preserving the consistent signals. The number of stacks quadruples with each graph from top left to bottom right, halving the level of the noise each time

Continuous seismic data was recorded for 33 hours. Unfortunately, the Y-component of the SARS5 sensor was not connected properly in this measurement, and the data from it was useless. The X-component's data was divided into $0.333 \mathrm{~s}$ intervals, each containing a separate measurement. These 350,000 
measurements were then used to produce a super-stack for the 33 hour period; this signal - the result of stacking $35 \mathrm{~GB}$ of data (Figure 4).

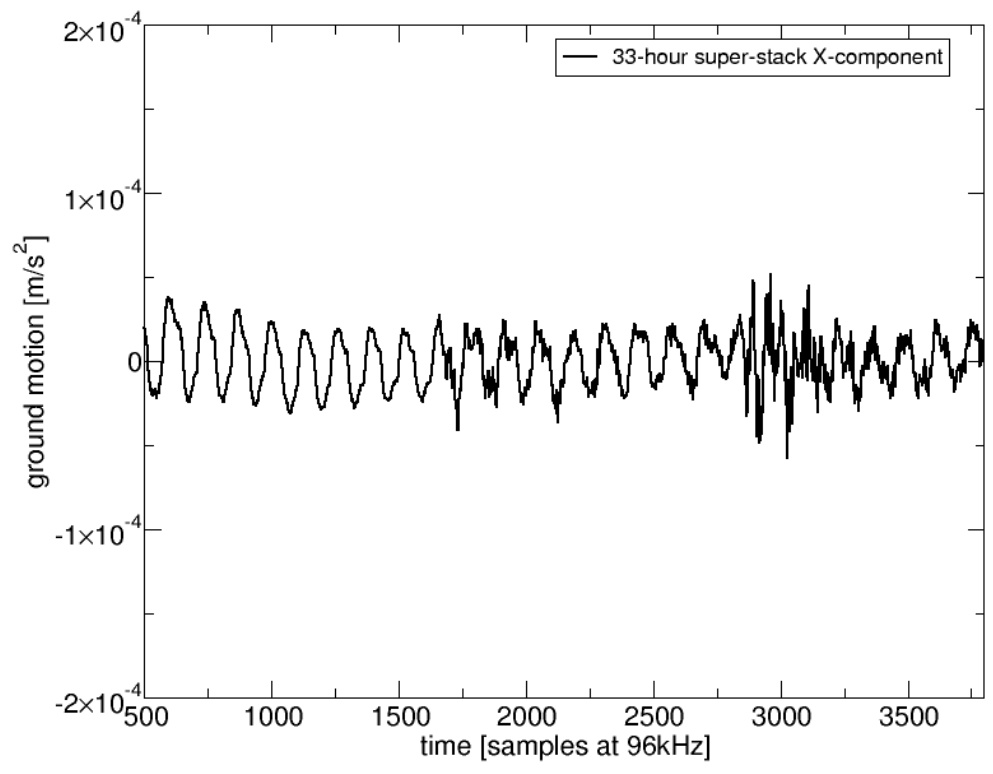

Figure 4 A super-stacked signal produced using 350,000 measurements of the pulsed piezoelectric source. The strong $720 \mathrm{~Hz}$ harmonic signal is the result of cross-talk across the data acquisition unit - it is the water column resonance recorded by the geophone $1 \mathrm{~m}$ above the source, attenuated by $58 \mathrm{~dB}$. High frequency arrivals are visible at about 1,750 and 2,800 samples

After a high-pass filter has been applied, the resulting signal reveals seismic wave arrivals corresponding to velocities of $6,400 \mathrm{~m} / \mathrm{s}$ and $3,640 \mathrm{~m} / \mathrm{s}$ (Figure 5). The P-wave velocity in the part of the mine is between $5,225 \mathrm{~m} / \mathrm{s}$ (in the footwall quartzite) and $6,630 \mathrm{~m} / \mathrm{s}$ (in the hangingwall lava), and so $6,400 \mathrm{~m} / \mathrm{s}$ is a reasonable value. The later dominant arrival corresponds to a velocity of $3,640 \mathrm{~m} / \mathrm{s}$. This appears to be an S-wave arrival, which is expected to have velocities between $3,000 \mathrm{~m} / \mathrm{s}$ (quartzite) and 3,800 m/s (lava) in this mine.

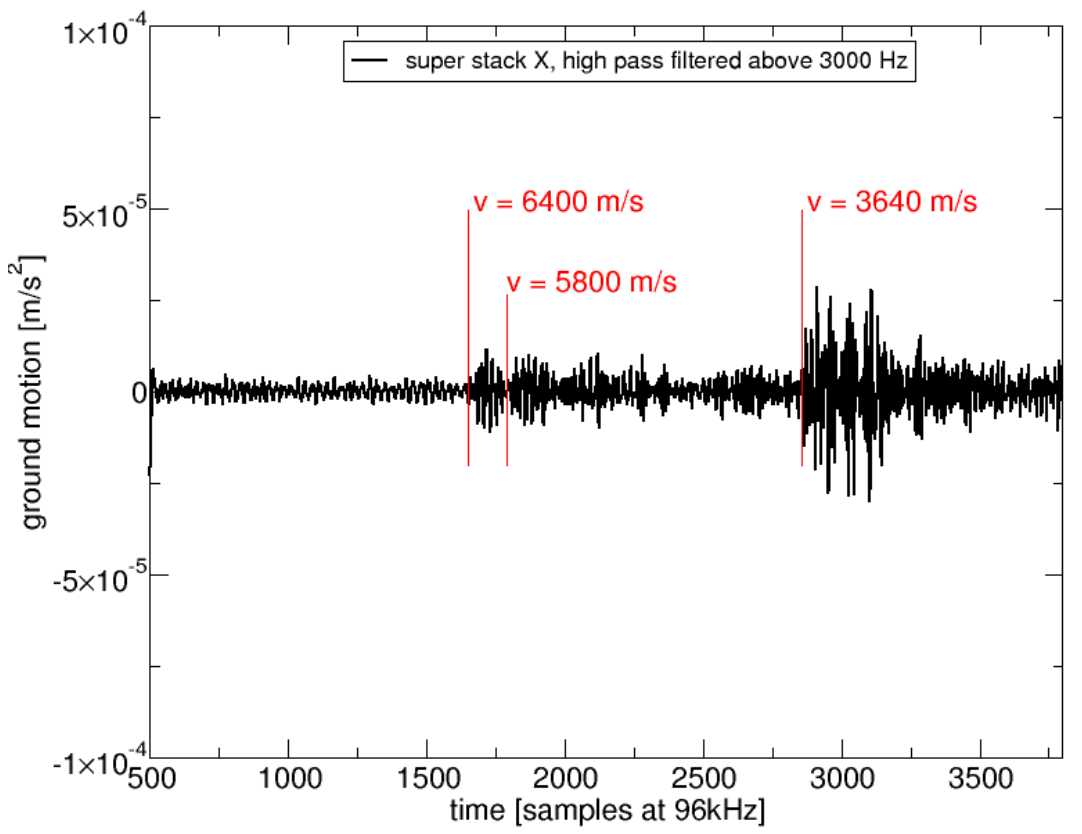

Figure $5 \mathrm{Filtered}$ and super-stacked signal recorded by the X-component of the sensor $107 \mathrm{~m}$ away from the source. Clear arrivals correspond to seismic velocities of $6,400 \mathrm{~m} / \mathrm{s}$ and 3,640 
$\mathrm{m} / \mathrm{s}$, and to a lesser extent $5,800 \mathrm{~m} / \mathrm{s}$. This corresponds to the expected $\mathrm{P}$ - and $\mathrm{S}$-wave velocities in this area

The appearance of an S-wave is unexpected but welcome. Theoretically, a cylindrically symmetric explosive source does not radiate $S$-waves in a radial direction in a homogeneous medium. However, we are most likely observing the effect of a P-S conversion at the borehole wall. It is very convenient to be recording this wave, as a simultaneous measurement of both P- and S-wave velocities yields an estimate of the Young's modulus $E$ and Poisson ratio $v$ through the expressions:

$$
\begin{gathered}
\mu=\rho V_{S}^{2} \\
\lambda=\rho V_{P}^{2}-2 \mu
\end{gathered}
$$

where $\mu$ and $\lambda$ are the Lamé parameters, $K=\lambda+\frac{2 \mu}{3}$ is the bulk modulus and

$$
\begin{gathered}
E=\frac{9 K \lambda}{3 K+\mu} \\
v=\frac{\lambda}{2(\lambda+\mu)}
\end{gathered}
$$

The same data analysis procedure was applied to five hours of recorded data from the X-component of the SARS6 sensor $206 \mathrm{~m}$ from the source. The resulting signal (Figure 6) still displays the same P-and S-wave arrivals, arriving later in accordance with the increased distance. Now the P-wave amplitude is stronger than the S-wave's, as expected from the sensor-source geometry.

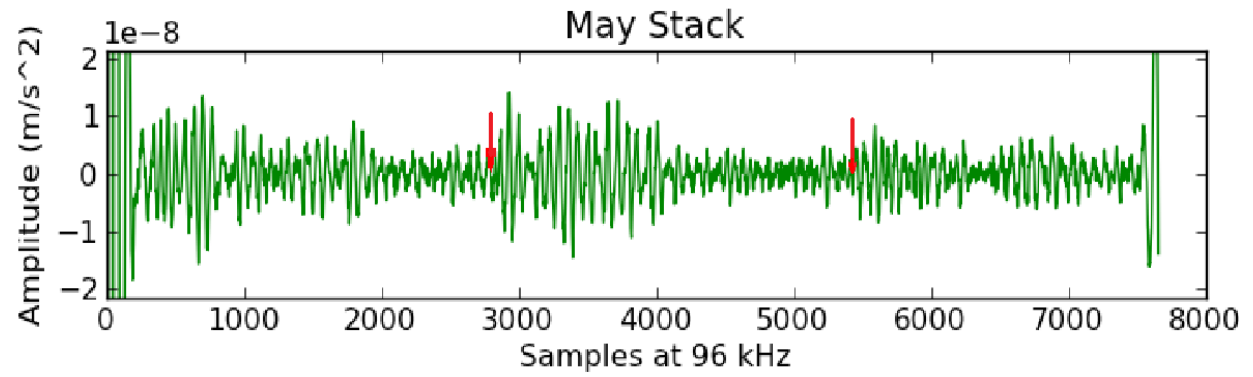

Figure 6 High-pass filtered super-stack five hours of SARS6 data. The clear P- and S-wave arrivals observed at the SARS5 sensor (107 $\mathrm{m}$ from the source) are also visible at the SARS6 sensor (206 $\mathrm{m}$ from source), indicated by red arrows. As expected from the sensor-source geometry, this signal - recorded by the X-component of SARS6 - shows a stronger P-wave than S-wave (reproduced from Harris (2011))

If we compare hourly stacks against the 33 hour super-stack for the $106 \mathrm{~m}$ sensor, we can see how velocity changes with time (Figure 7). If the underlying state (fracture density and stresses) of the rock was constant, then this scatter would be a measure of the accuracy of our velocity variations estimates - about $5 \times 10^{-4}$ in this case. However, the large deviation from the mean following blasting of panels about $100 \mathrm{~m}$ from this source-sensor ray at 19:25 hrs this day is suggestive of a sustained stress change induced by blasting that diffuses into the surrounding rock mass and is dissipated by relaxation after about 12 hours. Note that stress increase is the only mechanism to explain an increase in seismic velocity. This effect is similar to what was reported in Lynch (2010) following undercut blasting near the experiment at El Teniente mine.

The sharp drop and recovery in seismic velocity between 03:00 and 05:00 hrs may be related to the largest seismic event to occur in this vicinity during these measurements: a magnitude 1.4 event at 03:48 hrs, located about $150 \mathrm{~m}$ from the source-sensor ray. 


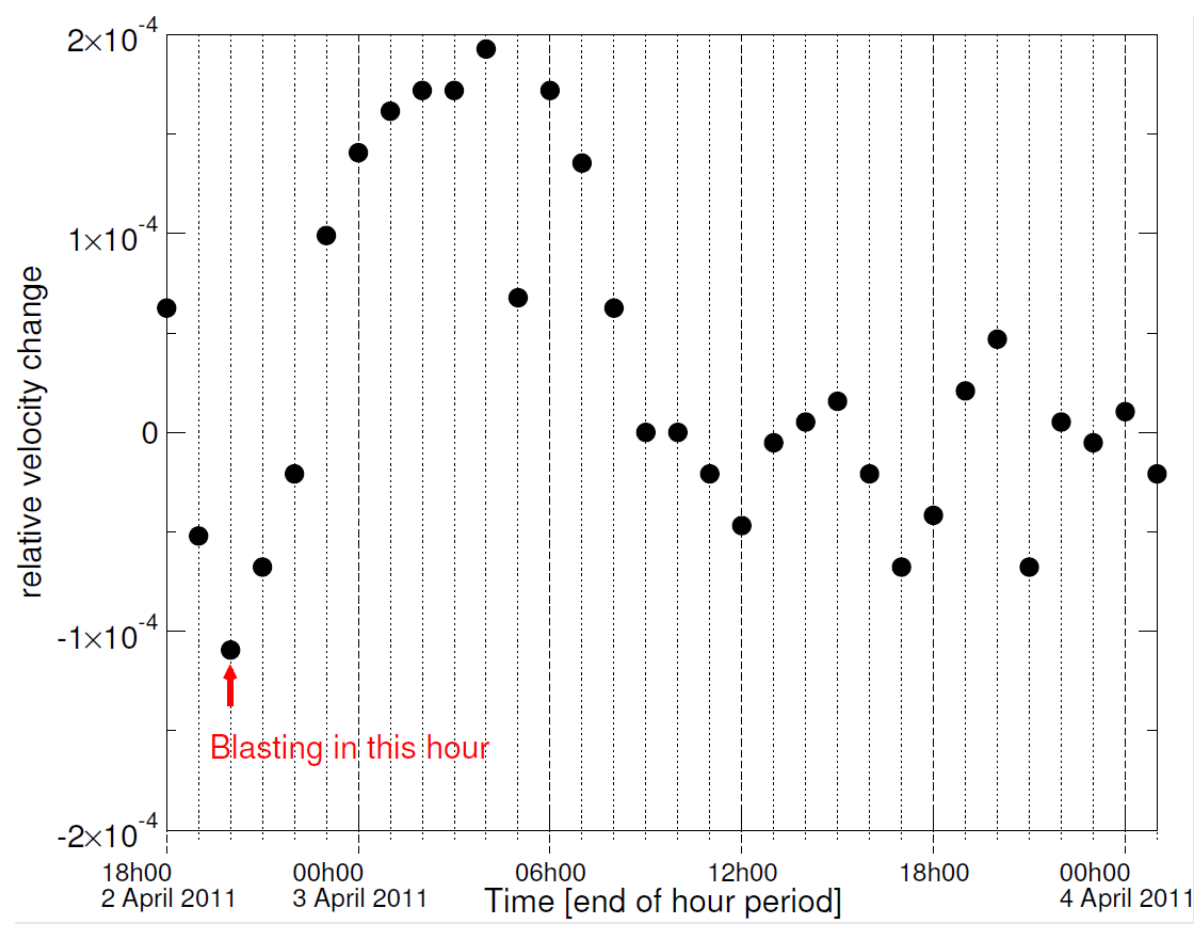

Figure 7 Seismic velocity changes extracted from comparison of the hourly stacks to the 33 hour super-stack. It is difficult to estimate the accuracy of these measurements, as the underlying stress change is expected to change following panel blasting $100 \mathrm{~m}$ away at 19:25. The increase in seismic velocity for 12 hours following blasting is suggestive of a temporarily increased stress, and is similar to what was reported by Lynch (2010)

\section{Experiment at Ridgeway Mine, Australia}

The Mponeng measurements were carried out by recording both the source pulse and the distant arrival on the same data acquisition unit. This set-up is required in order to identify the correct pieces of seismogram to stack. However, this arrangement limits the range of the experiment to a few hundred meters (the length of sensor cable) and produces massive datasets, which makes it impractical to do routinely.

In order to address this limitation, a pre-stacking algorithm was implemented within the embedded code of a GS seismic data acquisition unit. The DAQ is digitally time synchronised to the source driver to within $10^{-6} \mathrm{~s}$, and begins to record a seismogram 10 milli-seconds before the source is pulsed three times. Then the 1 -second seismogram is added to a stack buffer and a new seismogram starts. Every 5 minutes the 1 -second stack, consisting of 300 stacked seismograms, is transmitted to the central site and recorded. This pre-stacking algorithm reduces the data by a factor 300 , allowing the data to be transmitted in real-time to a server anywhere in the world. This arrangement also means the source driver and the data acquisition stations can be connected only by standard long-range digital communications.

To test this, a source and some triaxial sensors were installed the Halo area of the Ridgeway sublevel caving mine in NSW, Australia. The source is powered by a new version of the source driver, and begins the 3-pulse sequence $10 \mathrm{~ms}$ after a GPS pulse-per-second (PPS) signal is received. A data acquisition $100 \mathrm{~m}$ away receives the same PPS signal and begins to record the 1-second seismogram. If we use the closest sensor, installed $5 \mathrm{~m}$ from the source, we can record continuous data and stack it manually, in order to compare it to a 5 minute pre-stack. Figure 8 shows one of the 5 minute pre-stacks, and Figure 9 shows how the signal-to-noise (SNR) ratio grows with the manual stacks, compared with the 5 minute pre-stack SNR. 


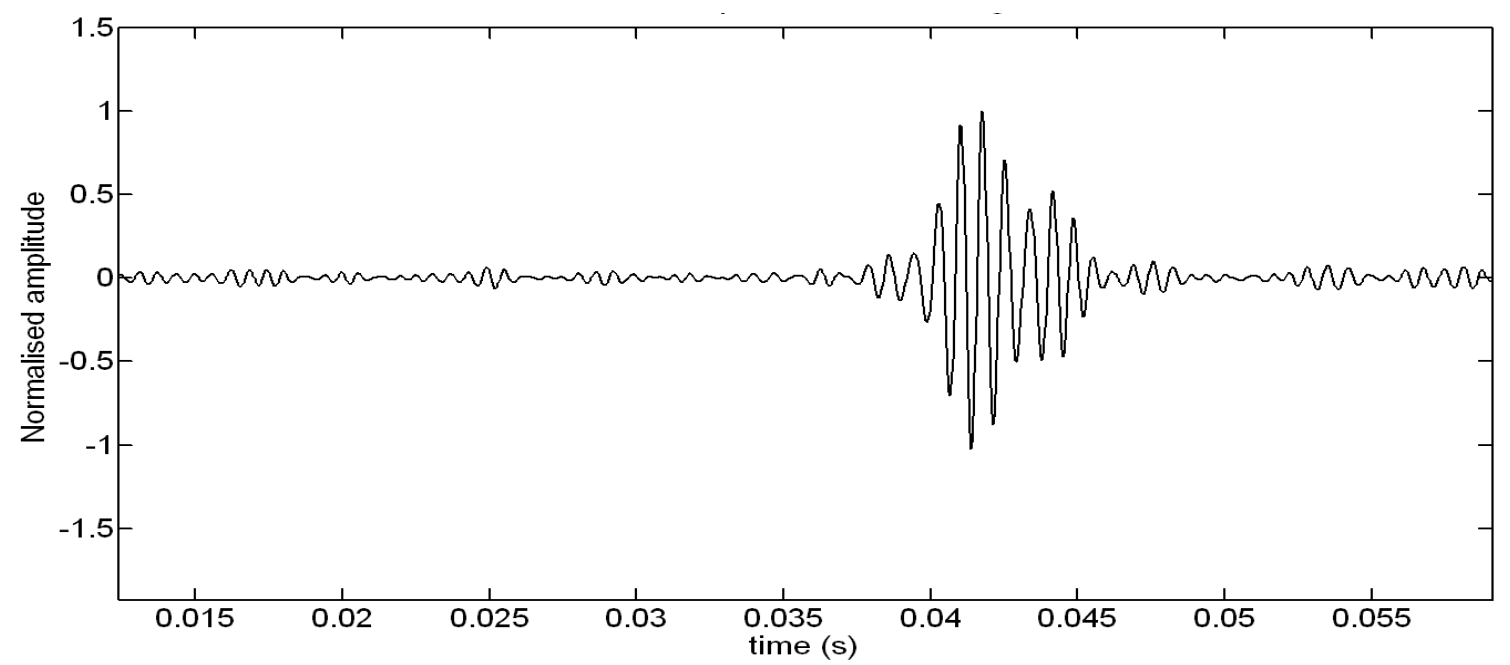

Figure 8 One of the 5 minute pre-stacks reported by the GS station monitoring the geophone installed $5 \mathrm{~m}$ from the source

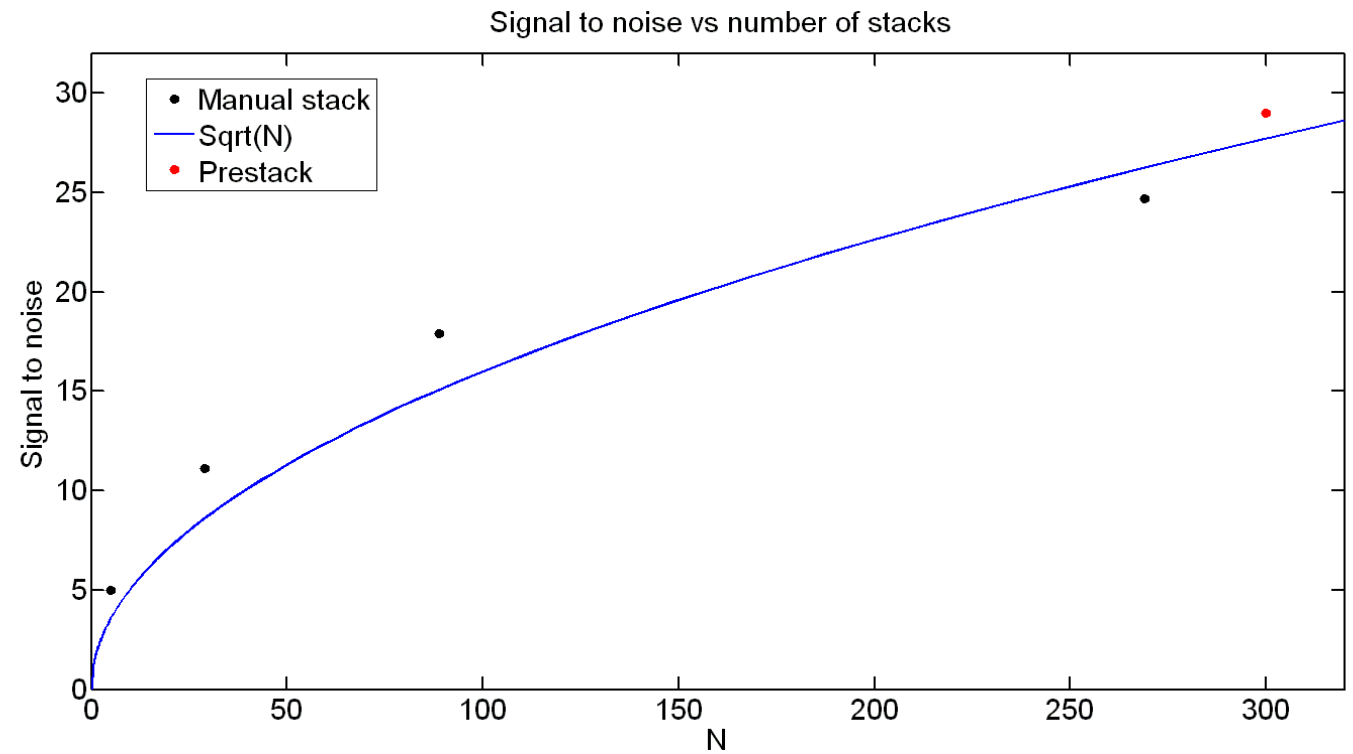

Figure 9 The signal-to-noise ratio obtained by the embedded pre-stacking algorithm (top right dot) compared with what we obtain by laboriously stacking the individual seismograms (dots) and what is expected theoretically if the noise is random and uncorrelated ( $\mathrm{ine}$ )

\section{$4 \quad$ Observations}

The measurements at Mponeng mine confirmed that massive stacking can be applied in working mine environments to extract signals produced continuously by a piezoelectric source. These stacked seismograms can be used to estimate seismic velocity variations on an hourly basis, and the 33 hour recording period reveals a period of increased seismic velocities following panel blasting in the vicinity.

The Mponeng experimental methodology used continuous seismic data and a sensor close to the source to obtain the exact time of the pulse. The massive amounts of data generated this way $(\sim 1 \mathrm{~GB} / \mathrm{sensor} / \mathrm{hour})$ makes this approach cumbersome for an experiment, and impractical (if not impossible) on a routine basis. A pre-stacking algorithm has been written for the embedded code running on the seismic station. This code allows stacking of seismograms to be achieved within the seismic station, and the pre-stacked signal is recorded every five minutes, resulting in factor 300 reduction in recorded data. Pre-stacking has been successfully demonstrated at Ridgeway mine, where a larger experiment is currently underway. 
With these results, and the results of previous work at El Teniente Mine, Active Seismic Monitoring currently has the following parameters:

- Piezoelectric borehole seismic source powered by high-voltage, time synchronised driver.

- Signals detectable at ranges of at least 200 m; scaling suggests even larger distances may be possible: $500-1,000 \mathrm{~m}$ ?

- Both P- and S-waves generated, which means effective medium measurements may be possible.

- Accuracy of seismic velocity variation measurements between $10^{-4}$ and $10^{-5}$, which appears to be sufficient to detect blasting-induced changes in local stress $100 \mathrm{~m}$ away.

- Pre-stacking within the seismic station, and storage of the resulting data on the central computer. This can be done at the same time as normal passive seismic monitoring of fracturing within the rock mass, which means that Active Seismic Monitoring comes at no cost to the normal monitoring activities.

\section{Acknow l edgements}

The author would like to gratefully acknowledge the support of two IMS Patrons: AngloGold Ashanti (particularly Shaun Murphy, Corne Moller and Gideon Potgieter) and Newcrest (particularly Dr Geoff Capes, Rob Lowther and Joe Emmi). Mark Green and Gerrit Olivier of IMS contributed to this work.

\section{References}

Birch, F. (1960) The velocity of compressional waves in rocks to 10 kilobars, part 1, in Journal of Geophysical Research, Vol. 65, pp. 1083-1102.

Birch, F. (1961) The velocity of compressional waves in rocks to 10 kilobars, part 2, in Journal of Geophysical Research, Vol. 66, pp. 2199-2224.

Boadu, F.K. and Long, L.T. (1996) Effects of fractures on seismic-wave velocity and attenuation, in Geophysical Journal International, Vol. 127, pp. 86-110.

Crampin, S. and Zatsepin, S.V. (1997) Modeling the compliance of crustal rock II - Response to temporal changes before earthquakes, in Geophysical Journal International Vol. 129, pp. 495-506.

De Fazio, T.L., Aki, K. and Alba, J. (1973) Solid earth tide and observed change in the in situ seismic velocity, in Journal of Geophysical Research, Vol. 78, pp. 1319-1322.

Gladwin, M.T. (1977) Simultaneous Monitoring of Stress and Strain in Massive Rock, Pageoph Vol., p. 115.

Harris, J. (2011) In-mine rock mass characterisation using Active Seismic Monitoring, BSc (Hons) thesis, University of Tasmania.

Hudson, J.A. (1981) Wave speeds and attenuation of elastic waves in material containing cracks, in Geophysical Journal International, Vol. 64, pp. 133-150.

Leary, P.C., Malin, P.E., Phinny, R.A., Brocher, T. and Voncolln, R. (1979) Systematic monitoring of millisecond travel time variations near Palmdale, California, in Journal of Geophysical Research, Vol. 84, pp. 659-666.

Lynch, R.A. (2010) Passive and Active Seismic Monitoring in Mines, keynote address at Deep and High Stress Mining Seminar 2010, Santiago, Chile.

Niu, F., Silver, P.G., Daley, T.M., Cheng, X. and Majer, E.L. (2008) Preseismic velocity changes observed from active source monitoring at the Parkfield SAFOD drill site, in Nature, Vol. 454, 10 July 2008.

Nur, A. (1971) Effects of stress on velocity anisotropy in rocks with cracks, in Journal of Geophysical Research, Vol. 76, pp. 2022-2034.

Nur, A. and Simmons, G. (1969) The effect of saturation on velocity in low porosity rocks, Earth Planetetary Science Letters, Vol. 7, pp. 183-193.

Ogasawara, H., Sato, S., Nishii, S., Sumitomo, N., Ishii, H., lio, Y., Nakao, S., Ando, M., Takano, M., Nagai, N., Ohkura, T., Kawakata, H., Satoh, T., Kusunose, K., Cho, A., Mendecki, A.J., Cichowicz, A., Green, R.W.E. and Kataka, M.O. (2002) Semi-controlled experiments in South African deep gold mines, The Journal of the South African Institute of Mining and Metallurgy, May/June 2002, pp. 243-250.

Reasenberg, P. and Aki, K. (1974) A precise, continuous measurement of seismic velocity for monitoring in situ stress, in Journal of Geophysical Research, Vol. 79, pp. 399-406.

Sano, O., Hieda, K., Hirano, K., Hirano, T., Ishii, H., Hirata, Y., Matsumoto, Y.S. and Yamauchi, T. (1999) Stress-sensitivity of the sound velocity at Kamaishi mine, paper presented at Seismological Society of Japan 1999 Fall Meeting, Sendai, Japan, 1999.

Scholz, C.H. (1968) Microfracturing and the inelastic deformation of rock, I: Compression in Journal of Geophysical Research, Vol. 73, pp. 1417-1432. 
Silver, P.G., Daley, T.M., Niu, F. and Majer, E.L. (2007) Active source monitoring of crosswell seismic traveltime for stress-induced changes, in Bulletin of the Seismological Society of America, Vol. 97, pp. 281-293 (2007).

Walsh, J.B. (1965) The effect of cracks on the compressibility of rock, in Journal of Geophysical Research, Vol. 70, pp. 381-389.

Yamamura, K., Sano, O., Utada, H., Takei, Y., Nakao, S. and Fukao, Y. (2003) Long-term observation of in situ seismic velocity and attenuation, in Journal of Geophysical Research, Vol. 108, No. B6, 2317 p.

Yukutake, H., Nakajima, T. and Doi, K. (1988) In situ measurements of elastic wave velocity in a mine, and the effects of water and stress on their variation, in Tectonophysics, Vol. 149, pp. 165-175. 